\title{
$K D R$ gene silencing inhibits proliferation of A549cells and enhancestheir sensitivity to docetaxel
}

\author{
R. Wei and J.-P. Zang \\ Department of Respiratory Medicine, People's Hospital of Zhengzhou, \\ Zhengzhou, China \\ Corresponding author: J.-P. Zang \\ E-mail: zangjping@163.com \\ Genet. Mol. Res. 14 (4): 14782-14789 (2015) \\ Received May 11, 2015 \\ Accepted July 22, 2015 \\ Published November 18, 2015 \\ DOI http://dx.doi.org/10.4238/2015.November.18.43
}

\begin{abstract}
We investigated the effects of kinase-domain insert containing receptor $(K D R)$ gene silencing on the proliferation of A549 cells and their sensitivity to docetaxel. After designing and synthesizing the KDR siRNA sequence, the sequence was transfected into A549 cells using Lipofectamine 2000. The expression of KDR mRNA and protein after $K D R$ gene silencing was detected by reverse transcription-polymerase chain reaction and western blotting; A549 cell cycle was detected by flow cytometry. An MTT assay and colony formation was performed to determine the sensitivity of A549 cells to docetaxel after KDR gene silencing. After 48-h KDR gene silencing, KDR gene and protein expression significantly decreased $(P<0.05)$. A549 cell cycle was significantly arrested in G0/G1 phase, and the number of cells in $S$ phase was reduced; the difference was statistically significant $(P<0.05)$ in the $K D R$ gene silencing group, sensitivity of A549 cells to docetaxel showed a significant enhancement $(P<0.05)$. KDR siRNA can significantly silence $K D R$ gene and protein expression in A549 cells, inhibit the proliferation of A549 cells, and enhance their sensitivity to docetaxel.
\end{abstract}

Key words: A549; Docetaxel; Kinase-domain insert containing receptor; Lung cancer; Proliferation; siRNA 


\section{INTRODUCTION}

The incidence of lung cancer is the highest worldwide. Its incidence is a multifactorial and progressive multi-step process, which has been a serious threat to human health (Feist et al., 2012; Liang 2014; Zhao et al., 2014a). In recent years, with the development of functional genomics and complete of the human genome project, it was discovered that tumor cell proliferation and migration was related with gene regulation, involved in the complex activation and deactivation of various genes, providing a new effective way to treat cancer (Kunert-Keil et al., 2011; Zhang et al., 2013; Ji et al., 2014). Studies have found (Lou et al., 2010; Xia et al., 2013) that kinasedomain insert containing receptor $(K D R)$ gene played an important role in the proliferation of lung adenocarcinoma cells, which can be used as targets for cancer therapy. KDR is a specific receptor that mediates the function of vascular endothelial growth factor in tumor angiogenesis, which is related to the hyperplastic state of malignancies. In this study, synthetic siRNA was transfected into A549 cells to analyze KDRsiRNA gene silencing efficiency; after KDR gene silencing, changes in the cell cycle and the effect on the sensitivity to docetaxel were evaluated to provide a theoretical basis for RNA interference KDR gene therapy of lung cancer or for the study of sensitizing drugs.

\section{MATERIAL AND METHODS}

\section{Materials}

A549 human lung cancer cells were purchased from Nanjing KGI Biotechnology Co., Ltd. (Nanjing, China). DMEM medium, Opti-MEM, G418, and trypsin were purchased from Gibco Company (Grand Island, NY, USA). Fetal bovine serum was purchased from Hangzhou Evergreen Company (Zhejiang, China). The liposomal transfection reagent Lipofectamine 2000 and TRIzol were purchased from Invitrogen (Carlsbad, CA, USA). The reverse transcriptionpolymerase chain reaction (RT-PCR) 2-step kit was purchased from TaKaRa (Shiga, Japan). The AMV reverse transcription kit was provided by the Chongqing Chuandong Chemical (Group) Co., Ltd. (Chongqing, China). The cDNA synthesis kit was from Toyobo Life Science (Osaka, Japan). RNA interference sequences were purchased from Guangzhou Ribio Co., Ltd. (Guangzhou, China). PCR primer sequences were chemically synthesized by Invitrogen. CCK-8 was from Shanghai Yan Bin chemical Company (Shanghai, China). Mouse anti-glyceraldehyde-3-phosphate dehydrogenase (GAPDH) monoclonal antibody and rabbit anti-human KDR polyclonal antibody were purchased from Abcam (Cambridge, UK). Horseradish peroxidase-labeled rabbit anti-goat IgG was purchased from Invitrogen. The polyvinylidene fluoride membrane was from Bio-Rad (Hercules, CA, USA). The BCA protein Assay Kit and RIPA protein lysate were purchased from Beijing SBS Gene Technology Co., Ltd. (Beijing, China). The electrochemiluminescence kit was purchased from Invitrogen. Matrigel gel was from BD Biosciences (Franklin Lakes, NJ, USA).

\section{Methods}

\section{siRNA design, synthesis, and transfection}

According to a previous study (Zhang et al., 2014), KDR siRNAand negative control scramble siRNA sequences were, respectively: 5'-GCCACCAUGUUCUCUAAUA'I'I'-3' (sense strand) 5'-UAUUAGAGAACAUGGUGGCAT-3' (antisense strand); 5'-UUCUCCGAACGUGUCACGU'IT-3' 
(sense strand), 5'-ACGUGACACGUUCGGAGAATI'-3' (antisense strand). Chemical synthesis was performed by the Shanghai Genepharma Company (Shanghai, China). A549 cells were cultured in $10 \%$ DMEM medium containing $10 \%$ fetal bovine serum in a $37^{\circ} \mathrm{C}, 5 \% \mathrm{CO}_{2}$, humidified incubator for closed culture, amplification, and passage. Cells in the logarithmic growth phase were used in this experiment. A549 cells were seeded on 6-well plates at a density of $2 \times 10^{5}$ cells/well; when the fusion rate was $80 \%$, the cells were transfected by the liposome method. The transfection was divided into 3 groups: cells transfected with pGenesil-1-KDR-siRNA vectors were included in the A549/KDR- siRNA group (experimental group); cells transfected with NC siRNA vectors were included in the A549/control group (control group); untransfected A549 cells were included in the A549 group (blank group). The RNA interference effect was detected at $48 \mathrm{~h}$ after transfection.

\section{Semi-quantitative RT-PCR}

The culture medium was discarded, and the cells were washed 3 times with phosphatebuffered saline (PBS); TRIzol was used to extract total RNA. Using the cDNA synthesis kit, cDNA was synthesized by reverse transcription to amplify the KDR and internal control GAPDH. The KDR upstream primer sequence was: 5'-CTGGCATGGTCTFCTGTGAAGCA-3', while the downstream primer was: 5'-AATACCAGTGGATGTGATGCGG-3'. The amplification products were 795 base pairs. The GAPDH upstream primer: 5'-CGTGGAAGGACTCATGACCA-3', while the downstream primer was: 5'-TCCAGGGGTCTTACTCCTTG-3'. The amplification product was 509 base pairs. PCR conditions were as follows: denaturation at $94^{\circ} \mathrm{C}$ for $2 \mathrm{~min}$, denaturation at $94^{\circ} \mathrm{C}$ for $45 \mathrm{~s}$, annealing at $62^{\circ} \mathrm{C}$ for $1 \mathrm{~min}$, extension at $72^{\circ} \mathrm{C}$, for a total of 35 cycles, with a final extension $8 \mathrm{~min}$ at $72^{\circ} \mathrm{C}$. The samples were analyzed by $2 \%$ agarose gel electrophoresis at $100 \mathrm{~V}$ for 45 $\min$. An automated imaging and analysis system was used to analyze the gel, and KDR/GAPDH absorbance values were considered to indicate the relative levels of mRNA expression.

\section{Western blotting}

The culture medium was discarded and cells were washed 3 times with PBS; total protein was extracted, and the protein concentration was determined using the BCA method. A total of $50 \mathrm{mg}$ protein was mixed with $20 \mu \mathrm{L}$ deionized water. An equal volume of $2 \mathrm{X}$ sample buffer was added before denaturation at $99^{\circ} \mathrm{C}$ for $10 \mathrm{~min}$. After centrifugation, $50 \mathrm{mg}$ samples were separated by electrophoresis, electro-transferred onto a polyvinylidene fluoride membrane, and sealed in blocking solution for $1 \mathrm{~h}$. Membranes were incubated with KDR primary antibody (1:1000) at $4^{\circ} \mathrm{C}$ overnight. Tris-buffered saline containing Tween 20 membrane-washing was performed 3 times for 5 min each time; 1:5000 horseradish peroxidase labeled secondary antibody and GAPDH were added and the samples were incubated at $37^{\circ} \mathrm{C}$ for $2 \mathrm{~h}$. After washing with PBS, samples were detected using electrochemiluminescence. X-ray film was exposed and photography was performed using a UVI gel imaging system (UVItech, Ltd., Cambridge, UK); gray values of bands were analyzed using Image-Pro Plus 7.0 software (Media Cybernetics, Inc., Rockville, MD, USA), with $\mathrm{KDR} / \mathrm{GAPDH}$ indicating the relative expression of KDR.

\section{Flow cytometry to detect cell cycle}

Cells in each group were collected and washed twice with cold PBS; next, the cells were precipitated and mixed with $70 \%$ cold ethanol $\left(4^{\circ} \mathrm{C}\right)$; after washing, the cell concentration was 
adjusted to $1 \times 10^{6} / \mathrm{L}$ with PBS, and then incubated with Tris- $\mathrm{HCl}$ buffer, $\mathrm{pH} 7.4$, containing $50 \mathrm{mg} /$ $\mathrm{mL}$ RNA enzyme for $30 \mathrm{~min}$. A total of $100 \mathrm{mg} / \mathrm{mL}$ propidium iodide was used to stain the DNA and incubated in the dark for $1 \mathrm{~h}$. The DNA content distribution of A549 cells was determined by flow cytometry, and the percentage of cells in each cycle was calculated.

\section{MTT assay to detect the sensitivity of A549 cells to docetaxel after KDR silencing}

After $48 \mathrm{~h}$ KDR siRNA transfection, A549 cells were collected for each group and seeded on 96-well plates; 3 wells were used for each sample. After adhesion, cells were cultured with 5 , 15,25 , or $50 \mu \mathrm{M}$ docetaxel. After culture for $48 \mathrm{~h}, 20 \mu \mathrm{L} 5 \mathrm{mg} / \mathrm{mL}$ MTT was added to each well; after another $4 \mathrm{~h}$ incubation, the supernatant was discarded and cells were incubated with $200 \mu \mathrm{L}$ $10 \%$ sodium dodecyl sulfate medium overnight. After shocking for $15 \mathrm{~min}$, the absorbance value (A value) at $570 \mathrm{~nm}$ was detected using an automatic microplate reader (Bio-Tek, Norcross, GA, USA). Inhibition rate $(\%)=(A$ control $-A$ experiment $) /(A$ control $-A$ blank $) \times 100 \%$.

\section{Plate cloning to detect the sensitivity of A549 cells to docetaxel after KDR silencing}

After 48 h KDR siRNA transfection, A549 cells were collected in each group and seeded on 6-well plates; 3 wells were used for each sample. After adhesion, cells were cultured with 5, 15, 25 , or $50 \mu \mathrm{M}$ docetaxel. After culture for 7 days, the medium was discarded and cells were washed twice with PBS. Next, the cells were fixed with $95 \%$ ethanol for 10 min, stained with Giemsa dye for 10 min, washed, and dried; according to the following equation, Quantity One software was used to calculate the inhibition rate of colony formation. Colony formation inhibition rate $(\%)=1$ - (number of clones in the experimental group / clone number in control group) x $100 \%$.

\section{Statistical analysis}

Measurement data were analyzed using SPSS 19.0 statistical software (SPSS, Inc., Chicago, IL, USA). Differences among groups were analyzed by univariate analysis of variance using the SPSS 11.5 windows software package. Continuous variables between 2 groups were compared using the $t$ test; continuous variables among groups were compared using analysis of variance and covariance correction. $P<0.05$ was considered statistically significant. Test level $\alpha=0.05$.

\section{RESULTS}

\section{KDR siRNA inhibited KDR mRNA expression}

RT-PCR of KDR mRNA expression showed that compared with the A549/control group (control group) and the A549 group (blank group), bands in the A549/KDR-siRNA group (observation group) were significantly narrower, and there were statistically significant differences between the A549/KDR-siRNA group and the A549/control group, the A549/KDR-siRNA group, and the A549 group (all $P<0.05$ ) (Figure 1).

\section{siRNA inhibited KDR protein expression}

Western blotting of KDR protein expression showed that compared with the A549/control 
group (control group) and the A549 group (blank group), bands in the A549/KDR-siRNA group (observation group) were significantly narrower, and there were statistically significant differences in the grey values (all $P<0.05)$ (Figure 2).
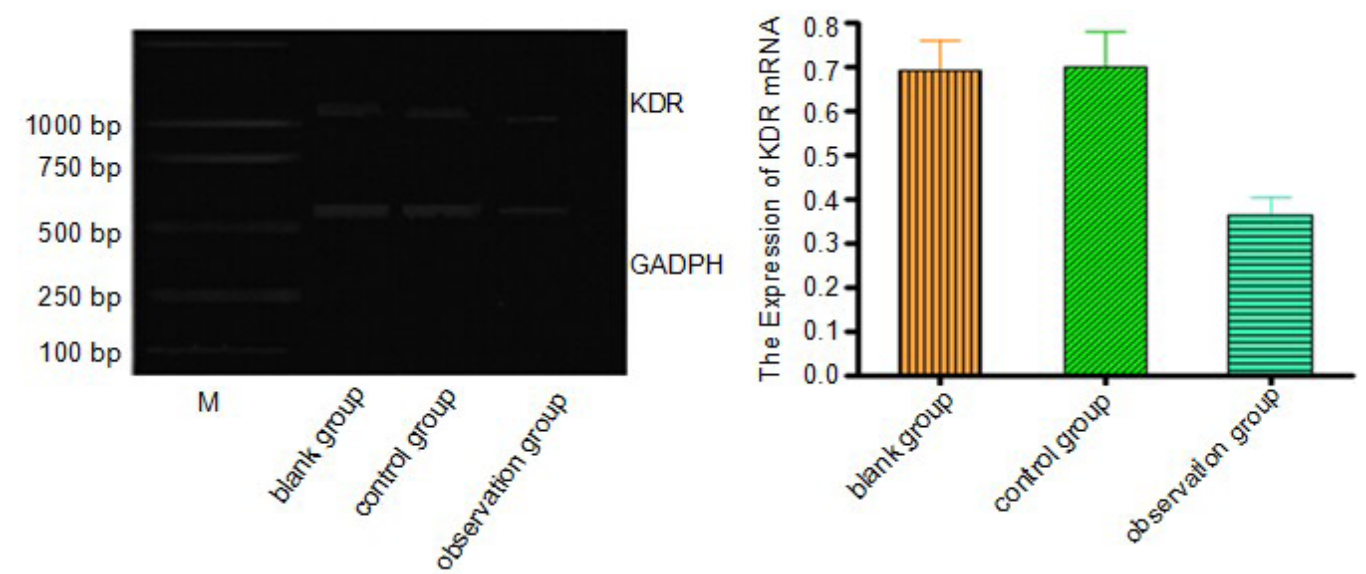

Figure 1. Detection of KDR mRNA expression in A549 cells by Reverse Transcription-PCR (RT-PCR).
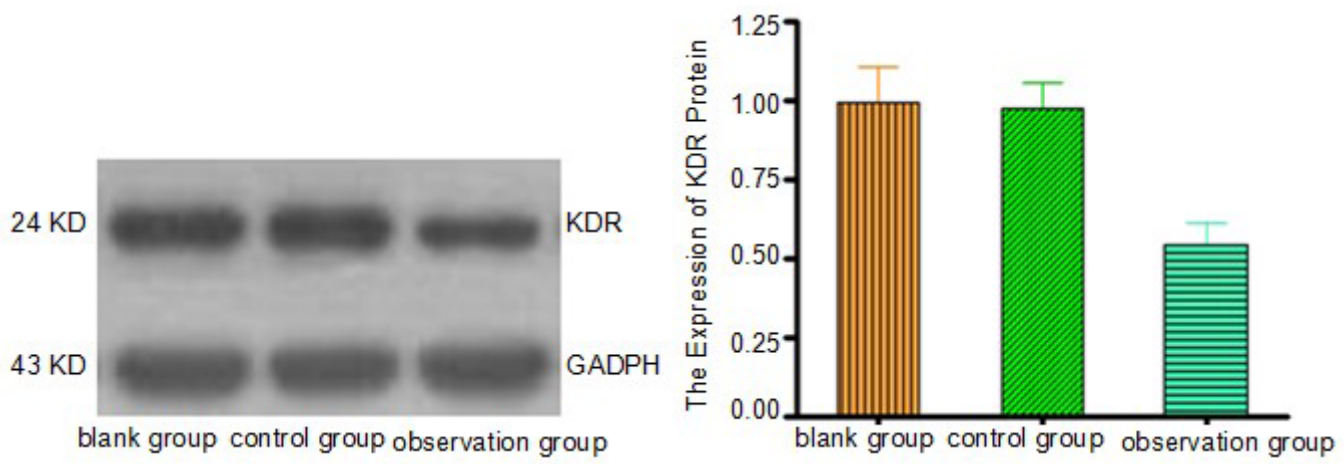

Figure 2. Detection of KDR protein expression in A549 cells by western blotting.

\section{Cell cycle detection by flow cytometry}

The A549 cell cycle was analyzed by flow cytometry; comparison of the A549/control group and A549 group showed that cells in G0/G1 phase increased slightly, while cells in S phase decreased slightly in the A549/KDR-siRNA group. There were statistically significant differences between the A549/KDR-siRNA group and the A549/control group, the A549/KDR-siRNA group, and the A549 group (all $P<0.05$ ). No significant changes were observed in $M$ phase and the difference was not statistically significant $(P>0.05)$, indicating a significant $S$ phase arrest in A549 cells (Table 1, Figure 3). 
Table 1. Effect of siRNA-inhibited KDR expression on A549 cell cycle (means $\pm s, N=3$ ).

\begin{tabular}{lccc}
\hline Group & G0/G1 & $\mathrm{S}$ & $\mathrm{G} / \mathrm{M}$ \\
\hline Blank & $52.8 \pm 2.8$ & $32.5 \pm 2.1$ & $8.3 \pm 1.2$ \\
Control & $52.6 \pm 3.6$ & $32.4 \pm 3.4$ & $8.6 \pm 1.3$ \\
Experiment & $70.5 \pm 6.3^{*}$ & $20.1 \pm 2.5^{\star}$ & $9.1 \pm 1.1$ \\
\hline
\end{tabular}

Compared with the blank and control groups, ${ }^{*} \mathrm{P}<0.05$.
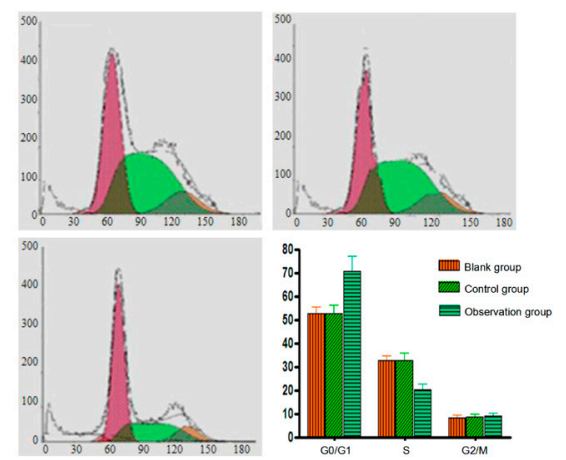

Figure 3. Flow cytometry for cell cycle detection.

\section{Increased sensitivity of A549 to docetaxel afterKDR interference}

The MTT assay showed that after siRNA-KDR transfection, the sensitivity of A549 cells to different concentrations of docetaxel was significantly improved. As drug concentration increased, the inhibition rate of docetaxel on A549 cells also increased in a dose-dependent manner. Compared with the control group, the sensitivity of A549 cells to different concentrations of docetaxel significantly increased $(P<0.05$, Table 2$)$.

Table 2. Inhibition rate of docetaxel on A549 cells detected by MTT assay after transfection (\%, means $\pm \mathrm{s}, \mathrm{N}=3$ ).

\begin{tabular}{lccr}
\hline Docetacel concentration $\mu \mathrm{M}$ & \multicolumn{2}{c}{ Inhibition rate (\%) } \\
\cline { 2 - 4 } & Blank group & Control group & Experimental group \\
\hline 0 & $15.43 \pm 1.32^{*}$ & $15.85 \pm 1.43^{*}$ & $26.65 \pm 2.76$ \\
6.25 & $22.54 \pm 1.54^{*}$ & $23.64 \pm 2.32^{*}$ & $38.32 \pm 2.52$ \\
12.5 & $31.43 \pm 2.05^{*}$ & $31.68 \pm 3.63^{*}$ & $51.27 \pm 3.87$ \\
25 & $41.87 \pm 1.43^{*}$ & $42.21 \pm 2.31$ & $75.71 \pm 4.54$ \\
50 & $51.45 \pm 3.65^{*}$ & $51.72 \pm 2.96^{*}$ & $91.45 \pm 3.21$ \\
\hline
\end{tabular}

${ }^{*} \mathrm{P}<0.05$ vs the experimental group.

\section{Effect on A549 colony formation}

The plate clone assay showed that after pgsiRNA-KDR transfection, the sensitivity of A549 cells to different concentrations of docetaxel was improved; the number of clones was significantly reduced and the colony formation inhibition rate was significantly increased $(P<$ 0.05). With increasing docetaxel concentration, the inhibition rate on cell colony formation also increased in a dose-dependent manner, indicating that the colony formation inhibitory rate in A549/KDR-siRNA group was significantly higher than that in the A549/control group and A549 group (all $P<0.05$, Table 3 ). 
Table 3. Inhibition rate of docetaxel on A549clone formation detected by plate clone after transfection (\%, means $\pm \mathrm{S}, \mathrm{N}=3$ ).

\begin{tabular}{lccc}
\hline Doxetacel $(\mu \mathrm{M})$ & \multicolumn{3}{c}{ Clone formation inhibition rate $(\%)$} \\
\cline { 2 - 4 } & Blank group & Control group & Experimental group \\
\hline 0 & $24.43 \pm 1.33^{*}$ & $25.22 \pm 1.55^{*}$ & $34.42 \pm 2.55$ \\
6.25 & $35.21 \pm 2.31^{*}$ & $34.55 \pm 1.46^{*}$ & $45.80 \pm 2.44$ \\
12.5 & $44.41 \pm 2.88^{*}$ & $45.33 \pm 2.55^{*}$ & $55.36 \pm 2.66$ \\
25 & $55.22 \pm 2.43^{*}$ & $54.68 \pm 1.22^{*}$ & $66.75 \pm 2.88$ \\
50 & $65.33 \pm 4.32^{*}$ & $67.43 \pm 3.42^{*}$ & $77.44 \pm 3.44$ \\
\hline
\end{tabular}

${ }^{*} \mathrm{P}<0.05$ vs the experimental group.

\section{DISCUSSION}

With an annually increasing incidence and gradually younger age of onset, lung cancer is becoming a serious problem (Yang et al., 2014; Xia and Zhou, 2014; Zhao et al., 2014b). Some studies showed that the incidence of tumors was related to several factors, including excessive cell proliferation, inhibition of apoptosis, and lack of death. These factors result in prolonged tumor cell survival in diseased tissues and destroy the alive-and-death balance in cells, which can lead to a larger number of surviving cells than dead cells and a net increase in the number of tumor cells. Therefore, methods for preventing the proliferation of tumor cells have gained increasing attention. In recent years, along with the rapid development of functional genomics and completion of the human genome project, gene therapy for cancer has been widely examined. The proliferation, invasion, and migration of lung cancer is involved in the structural and functional abnormalities of various genes, and thus identifying the role of key genes in the incidence of lung cancer is significant for the clinical diagnosis, treatment, and prevention of lung cancer.

The rapid development of RNA interference technologyprovides a foundation for tumor gene therapy, which has been widely examined in recent years (Chen et al., 2014; Lin et al., 2014; Pan et al., 2014). Previous studies found that KDR plays a key role in cell growth and differentiation, as KDR can act directly on tumor cells in an autocrine manner to promote cell proliferation and apoptosis. Based on the differences in KDR expression between the vascular endothelial cells in tumor and normal tissues, we designed and synthesized KDR siRNA sequences in this study, and A549 cells were transfected with Lipofectamine 2000 . We found that after $24 \mathrm{~h}$ transfection, the experimental group showed more pronounced growth inhibition, which was significantly enhanced at $48 \mathrm{~h}$. However, compared with the control group and the blank group, inhibition of plasmid on A549 cell growth was not observed in the experimental group.

In this study, KDR siRNA was transfected into A549 cells and the cell cycle was detected by flow cytometry; an MTT assay was performed to observe the sensitivity of A549 cells to docetaxel after KDR silencing. The results showed that the KDR gene was silenced; the cell cycle was arrested in G0/G1 phase. The number of cells in S phase was significantly reduced, and tumor growth was arrested. The sensitivity of A549 cells to docetaxel was significantly increased after KDR silencing, indicating that cell proliferation can be effectively and directly reduced, and the sensitivity of lung cancer cells to chemotherapy was strengthened. The results of this experiment showed that KDR gene silencing mediated by KDR siRNA can effectively inhibit cell proliferation and enhance the sensitivity of cancer cells to chemotherapy drugs, which can provide new research directions for the treatment or adjuvant treatment of malignant tumors. 


\section{Conflicts of interest}

The authors declare no conflict of interest.

\section{REFERENCES}

Chen M, Lu XZ and Zhang SY (2014). RNA interfere DNA repair improve the radiosensitivity of tumor cells. Chin. J. Cancer Biother. 21: 227-230.

Feist A, Lee R, Osborne S, Lane J, et al. (2012). Increased incidence of cutaneous squamous cell carcinoma in lung transplant recipients taking long-term voriconazole. J. Heart Lung Transplant. 31: 1177-1181.

Ji SQ, Zhao YQ, Han ZX, Ji SQ, et al. (2014). CD74 inhibits 786-O cell invasion in renal cancer by modulating CXCR4 expression. J. Mod. Urol. 111-113.

Kunert-Keil C, Steinmüller F, Jeschke U, Gredes T, et al. (2011). Immunolocalization of glycodelin in human adenocarcinoma of the lung, squamous cell carcinoma of the lung and lung metastases of colonic adenocarcinoma. Acta Histochem. 113: 798-802.

Liang X (2014). Chinese Academy of Medical Sciences Cancer Hospital from 2005 to 2013 first diagnosed with lung cancer characteristics of hospitalized patients. China Cancer 23: 376-380.

Lin SH and Liu Q (2014). RNA interference gene therapy technology and lung disease. Chin. J. Med. Educ. Res. 38: 905-908.

Lou XY, Sun HY and Liu XQ (2010). Vascular endothelial growth factor and its receptor KDR in peripheral lung cancer and its clinical significance. Cont. Med. 16: 28-29.

Pan YM, Zhang W, Ma W, et al. (2014). NS gene silencing RNA interference in human non-small cell lung cancer cell proliferation and apoptosis of A549. Chin. J. Cancer Biother. 21: 532-536.

Xia HG, Ye JF, Bai HY and Wang C (2013). Effects of cetuximab combined with celecoxib on apoptosis and KDR and AQP1 expression in lung cancer. ZhongguoFei Ai ZaZhi 16: 625-631.

Xia LM and Zhou YY (2014). rmhTNF- $\alpha$ combined with cisplatin inhibits proliferation of A549 cell line in vitro. Chin. Med. Sci. J. 29: 185-187.

Yang WT, Chen DL, Zhang FQ, Xia YC, et al. (2014). Experimental study on inhibition effects of the XAF1 gene against lung cancer cell proliferation. Asian Pac. J. Cancer Prev. 15: 7825-7829.

Zhang MS, Hu AH, QH, Sun Q, et al. (2013). Impact of STAT6 gene silencing on proliferation of human colon cancer cell HT-29. Med. J. Wuhan Univ. 34: 1-5, 49.

Zhang X, Ge YL, Zhang SP, Yan P, et al. (2014). Downregulation of KDR expression induces apoptosis in breast cancer cells. Cell. Mol. Biol. Lett. 19: 527-541.

Zhao J, Wang W, Shang LQ, Wang L, et al. (2014a). The comparison between haplo-chemotherapy and endostar added with chemotherapy for postoperative non-small cell lung cancer patients. Translat. Med. J. 19-21.

Zhao X, Yuan Y, Zhang Z, Feng X, et al. (2014b). Effects of shRNA-silenced livin and survivin on lung cancer cell proliferation and apoptosis. J. BUON 19: 757-762. 